\title{
OBJECTIVE DRIVEABILITY: INTEGRATION OF VEHICLE BEHAVIOR AND SUBJECTIVE FEELING INTO OBJECTIVE ASSESSMENTS
}

\author{
Isa Yassir Arafat Machmudi Isa ${ }^{1}$, Mohd Azman Zainul Abidin ${ }^{2}$ and \\ Shuhaimi Mansor ${ }^{2}$ \\ ${ }^{1}$ Homologation and Testing, Engineering Division, PROTON, \\ 40918 Shah Alam, Selangor, Malaysia \\ ${ }^{2}$ Faculty of Mechanical Engineering, Universiti Teknologi Malaysia, \\ 81310 Skudai, Johor, Malaysia \\ Email: yassirmi@proton.com
}

\begin{abstract}
Vehicle drivability is defined as the smoothness of a vehicle's operation at the will of a driver under all driving conditions. Currently, drivability evaluation is conducted through a subjective ratings-based test standard which is derived from established procedures. Human subjective rating can be inconsistent due to physical health conditions and individual preferences. This study is conducted to determine the possibility of using longitudinal acceleration to arrange an objective drivability assessment. Vehicle evaluation is conducted to determine the subjective vehicle drivability ratings of four drivability expert evaluators. A test vehicle was evaluated under different acceleration conditions to determine a subjective drivability rating. Vehicle low speed passing acceleration during pedal tip in is measured. A relationship between low speed passing acceleration and subjective drivability rating is established. An objective drivability assessment tool is successfully arranged using this relationship. A drivability rating can be generated using the tools, without the need for subjective evaluation by expert evaluators.
\end{abstract}

Keywords: Drivability; subjective rating; longitudinal acceleration; objective drivability assessment.

\section{INTRODUCTION}

Drivability is defined as the smoothness of vehicle operation, with the engine and power transmitting system under control of driver under all weather and driving conditions (Proton, 2009). Drivability determines whether the car behaves as per customer demands. Drivers have their own preferences and descriptions of how a vehicle should behave under specific conditions. For example, they want a vehicle that moves instantly when they press an accelerator pedal. Some drivers prefer a relaxed and progressive acceleration while others prefer a sportier, more sudden feel of acceleration. normal driver define drivability as both positive and negative perceptions (Schoeggl \& Ramschak, 2000). A positive perception includes feelings such as fast acceleration, good pedal response, precise gear shifts, and being easy to start and easy to drive. Most vehicle manufacturers conduct drivability tests through a subjective 10 point rating system, with 10 representing the best rating as described by (Cross, Thirard, Antoine, \& Dolcini, 2010; Palumbo, Amante, \& Andrea Ugo, May 2007; Yassir, 2008). An evaluation trip will be conducted for each developmental stage, to verify drivability performance. A group of drivability evaluators give individual subjective ratings for 
each drivability item based on certain test standards. Ratings are different from one evaluation to another. Subjective ratings are difficult to reproduce due to the different health conditions and environmental settings of the evaluators.

The traditional drivability development approach of giving subjective ratings during an actual driving evaluation lengthens time for development. After engine control unit (Neculita, Zagury, \& Bussière, 2007) changes, all evaluators must verify such changes in an evaluation trip and decide whether the subjective rating has improved. This iteration process takes a long time to complete. This study was conducted to determine the possibility of converting a vehicle's acceleration data into an objective drivability assessment. This involves integration of the vehicle's acceleration with a driver's subjective feeling. A series of evaluations and experiments were conducted to gather data from both driver and vehicle. The data was evaluated and integrated into one objective assessment. Some benefits of the study:

i. A consistent drivability rating every time, without influence of the driver's subjective feelings.

ii. Generalization of drivability evaluation. Everyone can conduct a drivability evaluation with valid test results, without depending on expert evaluators.

iii. Benchmarking and data collection: clear objective data about competitor's drivability levels.

iv. Shorter development times due to a shorter iteration sequence in ECU calibration tuning. Early involvement in early development stage.

This research will focus on establishing a drivability equation based on input from vehicle acceleration and the output of drivability ratings. The equation will be modeled in software, and integrated with data acquisition to form an objective drivability assessment tool.

\section{DRIVABILITY EVALUATION}

Proper procedure is essential in determining vehicle drivability ratings since driving involves many parameters such as those of the driver, vehicle conditions and road conditions. Customer perceptions of drivability are also important as it can become a unique selling point for a new model. Drivability can also improve overall quality, reduce physical prototypes and assist development efforts (AVL List, 2000). The SAE test standard (1993) categorizes accelerator pedal operation into four different levels as in Table 1. The description of the level depends on a manifold pressure reading. 'Light tip in' (LTI) is defined as an accelerator pedal operation that gives a manifold pressure reading of $40.4+0.6$ idle $\mathrm{kPa}$. For 'wide open throttle' (WOT), it is set at $101 \mathrm{kPa}$. Due to this set value, the procedure is difficult to follow. A pressure sensor is required in order to confirm the manifold pressure.

Table 1. Manifold pressure for each pedal position (SAE, 1993).

\begin{tabular}{ccc}
\hline No & Tip in & Manifold pressure \\
\hline 1 & LTI & $40.4+0.60$ idle kPa \\
2 & MTI & $60.6+0.40 \mathrm{kPa}$ \\
3 & HTI & $85.8+0.15$ idle kPa \\
4 & WOT & $101 \mathrm{kPa}$ \\
\hline
\end{tabular}


Dorey and Holmes (1999) defined drivability evaluations according to a combination of the tip in or back out maneuver based on rpm, vehicle speed and throttle position, as per Table 2. They consider low speed drivability as a critical evaluation factor. The procedure is a simulation of city driving conditions. The accelerator positions are defined as zero, quarter, half and full operation. The drivability procedure is easily followed. All vehicles operations are clearly defined based on gear position, rpm, vehicle speed, vehicle operating condition and throttle position. Every driver, especially those with less experience in drivability, can follow the procedure. The procedure is suitable for conducting market surveys or customer acceptance activities.

Table 2. Drivability evaluation procedure (Dorey \& Holmes, 1999).

\begin{tabular}{ccccc}
\hline Gear & $\mathrm{Rpm}$ & $\mathrm{Km} / \mathrm{h}$ & Maneuver & Throttle \\
\hline 1 & 1500 & 20 & Cruise to accelerate & Full \\
2 & 2000 & 30 & Decelerate to accelerate & Quarter \\
2 & 2000 & 30 & Decelerate to accelerate & Full \\
2 & 3000 & 45 & Decelerate to accelerate & Quarter \\
2 & 3000 & 45 & Decelerate to accelerate & Full \\
1 & 1500 & 20 & Cruise to decelerate & Zero \\
\hline
\end{tabular}

According to Schöggl et al. (2002) a WOT operation is used to determine vehicle characteristics. The test vehicle speed is maintained at $35 \mathrm{~km} / \mathrm{h}$ before full acceleration pedal input. Time is recorded until vehicle speed reaches $50 \mathrm{~km} / \mathrm{h}$. This simulates vehicle operation during overtaking maneuvers. A vehicle speed vs. time graph is plotted during acceleration maneuvers on a test vehicle as per Figure 1. Instantaneous acceleration is calculated and a sine wave graph recorded based on time domain.

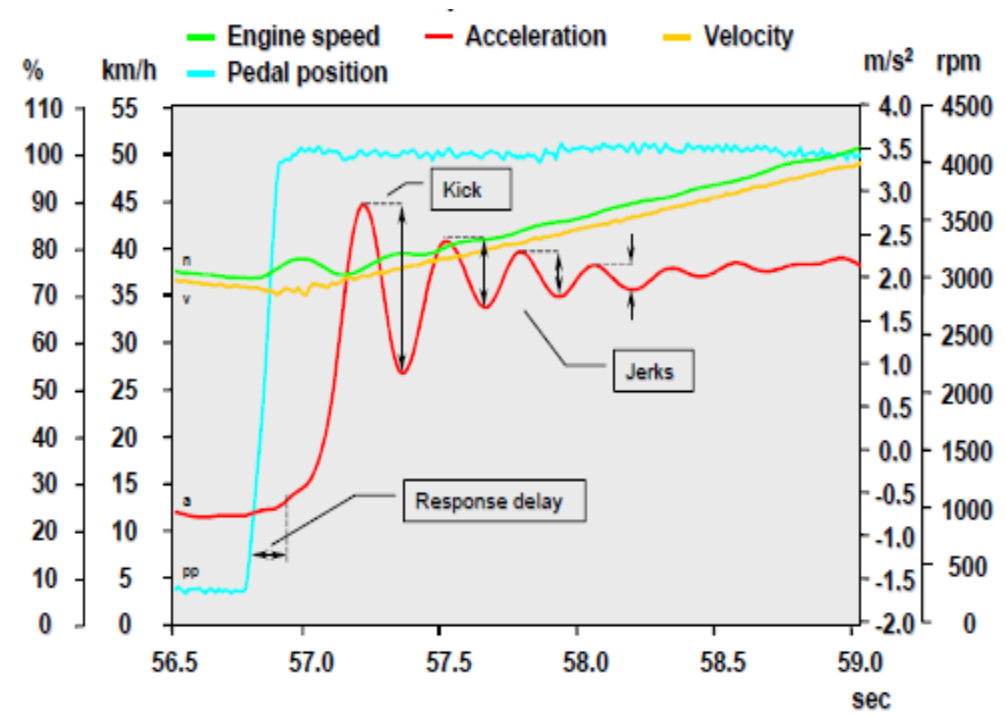

Figure 1. Acceleration behavior during WOT application (Schöggl et al., 2002).

From the graph plotted in Figure 1, longitudinal acceleration behaviors can be studied. Immediately after accelerator pedal operation, there may be a slight delay 
before the vehicle begins to move. This response delay is defined as the time measured between pedal activation and the first increment of acceleration. A vehicle immediately accelerates to its maximum acceleration before decelerating and repeat this sequence until steady state acceleration is achieved. The first decrease of longitudinal acceleration is defined as the 'kick'. The sequence of acceleration and deceleration until stable condition is defined as 'jerks'. Dorey and Holmes (1999) reported that peak acceleration is defined as the first acceleration peak after tip in as per Figure 2. The acceleration slowly stabilizes into steady state acceleration. This situation is measured as a damping ratio. Steady state acceleration is declared as the initial acceleration value.

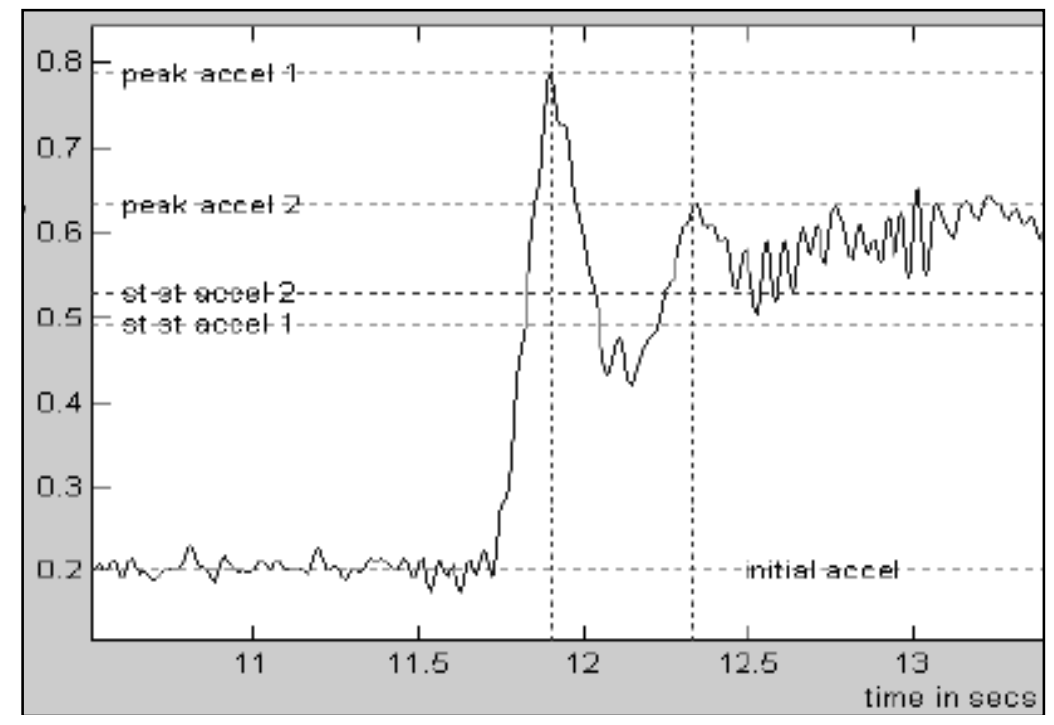

Figure 2. Acceleration behavior during tip in Dorey and Holmes (1999).

High noise acceleration data was captured during the test procedure. A noise filter must be introduced before determining the actual acceleration value. Due to noise, the steady state acceleration value might not be accurate for use in determining an objective drivability rating. Different approaches to determining acceleration value, such as using a longer sampling time can be proposed. With a longer sampling time, acceleration value should be stabilized. Little research has been conducted into determining a method of converting subjective drivability data into an objective drivability rating. Most researchers accept that there is a relationship between subjective drivability ratings given based on feeling with vehicle's behavior. According to (Dorey \& Holmes, 1999), a strong relationship between drivers and drivability items is shown by data trends. Data is gathered based on surveys, where the general public evaluate the same test vehicle. From the graph in Figure 3, a mathematical equation can be formed that represents the relationship of a subjective rating to a vehicle's behavior. Unfortunately, the actual mathematical equation cannot be presented. It is considered a trade secret, which is used internally within Ricardo's engineering control. A general hypothesis is suggested: there is clear indication that a driver's assessment is influenced by vehicle smoothness and speed of response. This gap will be addressed in the research. 


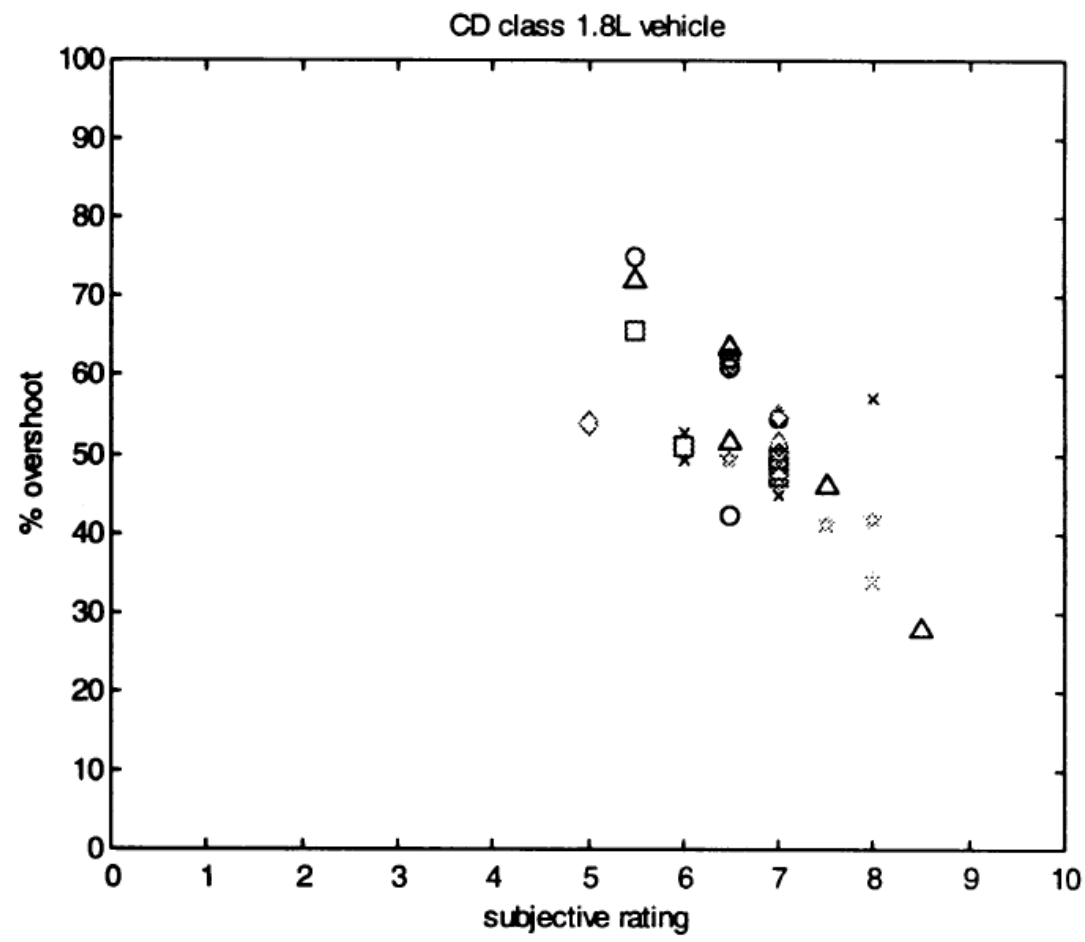

Figure 3. Relationship between vehicle's behavior and subjective rating (Doray \& Martin, 2000).

\section{METHODOLOGY}

A specific test parameter is set for this research. Low speed passing acceleration is described as a vehicle's acceleration behavior when overtaking at low speed. The test vehicle is maintained at a specific initial speed $(15 \mathrm{~km} / \mathrm{h})$ in $2^{\text {nd }}$ gear. Test drivers then applied the wide open throttle (WOT) operation. The test vehicle accelerated to a final speed $(25 \mathrm{~km} / \mathrm{h})$. The time taken to accelerate from $15 \mathrm{~km} / \mathrm{h}$ to $25 \mathrm{~km} / \mathrm{h}$ was measured. The reaction of vehicles in this procedure is represented in Figure 4.

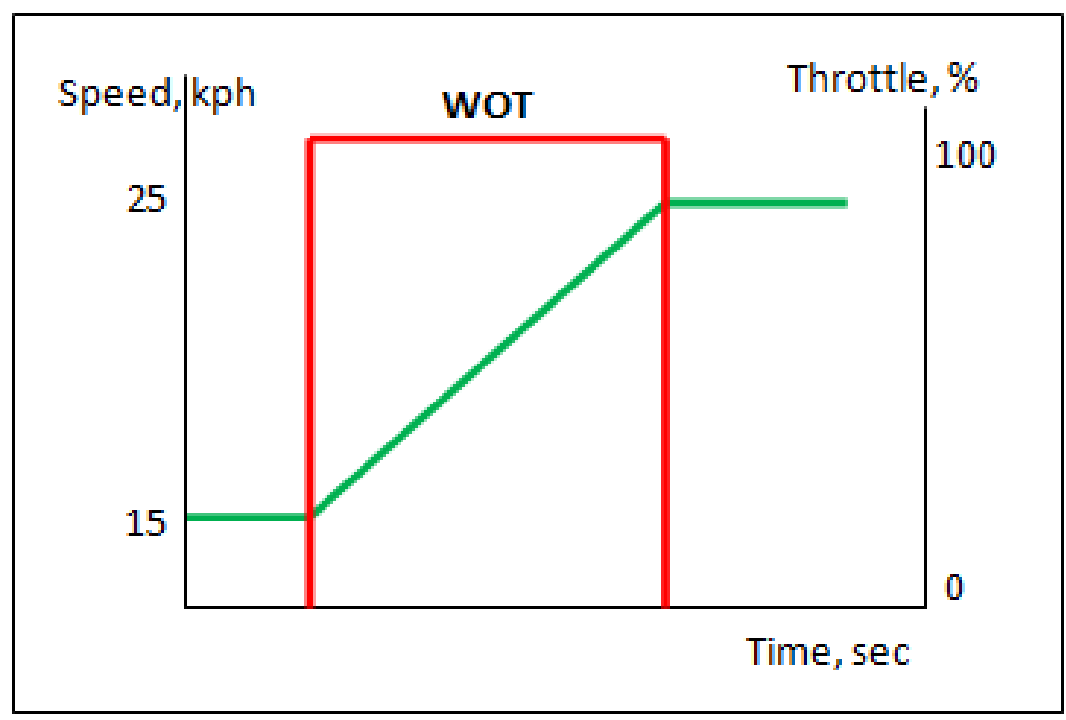

Figure 4. Low speed passing acceleration. 
The whole procedure is designed to replicate a vehicle's behavior during overtaking at low speed. Second gear was selected to simulate city driving conditions. The WOT operation represents a situation where maximum performance of a vehicle is required in order to overtake other vehicle. A Proton Model 1 was selected for the research. It was launched in 2010 and equipped with an intake air fuel module (IAFM) engine, an improvement over the original Campro's torque character. The long wheelbase, as indicated in Table 3, will minimize the effect of pitching movement during longitudinal acceleration. A manual gearbox ensures a continuous acceleration profile is generated without influence of a gear shift/ kick down. A mechanical load is applied to the vehicle in order to produce different passing acceleration characteristics by means of parking brake application in different settings so that the resistance at the rear wheel will reduce the passing acceleration rate.

Table 3. Proton Model 1 specification

\begin{tabular}{lc}
\hline \multicolumn{1}{c}{ Item } & Proton Model 1 \\
\hline Engine & $1598 \mathrm{cc}$ \\
Maximum power & $82 \mathrm{~kW} / 6500 \mathrm{rpm}$ \\
Maximum torque & 148Nm / 4000rpm \\
Transmission & F5M41 \\
Wheelbase & $2600 \mathrm{~mm}$ \\
Curb weight & $1200 \mathrm{~kg}$ \\
$0-100 \mathrm{~km} / \mathrm{h}$ acceleration & $11.5 \mathrm{sec}$ \\
\hline
\end{tabular}

Data was collected from the instrumentation using VBOX equipment for time and speed measurement. It has a measurement accuracy of $1 \%$ for acceleration and a 0.01 second error in time recording. The sensor was placed at the vehicle's center of gravity to minimize the effect of pitching during longitudinal acceleration. A subjective drivability rating was given to evaluate the vehicle's performance in the set procedure. The rating was given on a scale of 1 to 10 as per Table 4, with 10 representing the best rating. Ten points would be given if the evaluator feels that the vehicle has exceptional performance in an aspect of drivability. A rating of 7 is described as minor deficiencies which only an experienced driver would notice. Such a minor deficiency would not create customer complaints. A rating of 5 is described as marginal, and may cause a normal driver to make a complaint. If it involves safety or a situation where the test vehicle's maneuver tends to inspire a lack of confidence, a rating of 3 is given.

Table 4. Subjective rating definition (SAE, 1993).

\begin{tabular}{cl}
\hline Rating & \multicolumn{1}{c}{ Definition } \\
\hline 10 & Exceptional \\
9 & No deficiencies \\
8 & No significant deficiencies \\
7 & Minor deficiencies \\
6 & Obvious, but not objectionable problem \\
5 & Marginal \\
4 & Disturbing \\
3 & Lack of confidence \\
2 & Unreliable \\
1 & Unpredictable \\
\hline
\end{tabular}


In this research, test evaluators evaluated the test vehicle's behavior during low speed passing acceleration. Subjective assessment was based on how they feel during driving. Different acceleration levels set for the test vehicle give different feels. The evaluators will experience sluggishness and a lack of confidence if the vehicle's acceleration is slow. Fast acceleration will create a good performance feel. Excessive acceleration will tend to create an uncontrollable situation where the evaluators feel that there may be a tendency to hit another vehicle.

\section{RESULTS AND DISCUSSION}

Figure 5 shows acceleration and speed behavior during a drivability procedure. It shows that in second gear, the test vehicle's crawling speed is $13 \mathrm{~km} / \mathrm{h}$. Immediately after WOT application, there is significant increase in acceleration, before it stabilizes after approximately 1 second. The vehicle's speed increased in more linear pattern starting at $15 \mathrm{~km} / \mathrm{h}$. The acceleration immediately dropped once the WOT accelerator pedal was released. Time was recorded for the test vehicle's move from $15 \mathrm{~km} / \mathrm{h}$ to $25 \mathrm{~km} / \mathrm{h}$. Average acceleration was recalculated based on the speed difference. Although the initial acceleration played important role in determining the vehicle's low speed performance, it only happened in short time ( 0.2 seconds). The initial peak acceleration was below the steady state acceleration value. The steady state acceleration had more influence throughout the WOT operation. According to the evaluation, most evaluators provided the rating based on overall acceleration from $15 \mathrm{~km} / \mathrm{h}$ to $25 \mathrm{~km} / \mathrm{h}$. Low speed passing acceleration is one of critical drivability criteria in a car's development. It will determine a vehicle's reaction when the customer drives the vehicle for the first time. This will influence a customer's overall perception of the vehicles. A customer will immediately classify the vehicle as sluggish, responsive or sporty, according to this. Low speed passing acceleration is also related to safety. It will determine whether a car has sufficient acceleration during overtaking and low speed maneuvers such as at traffic lights and road junction crossings. From this research, the exact acceleration values that ensure best low speed passing acceleration can be determined.

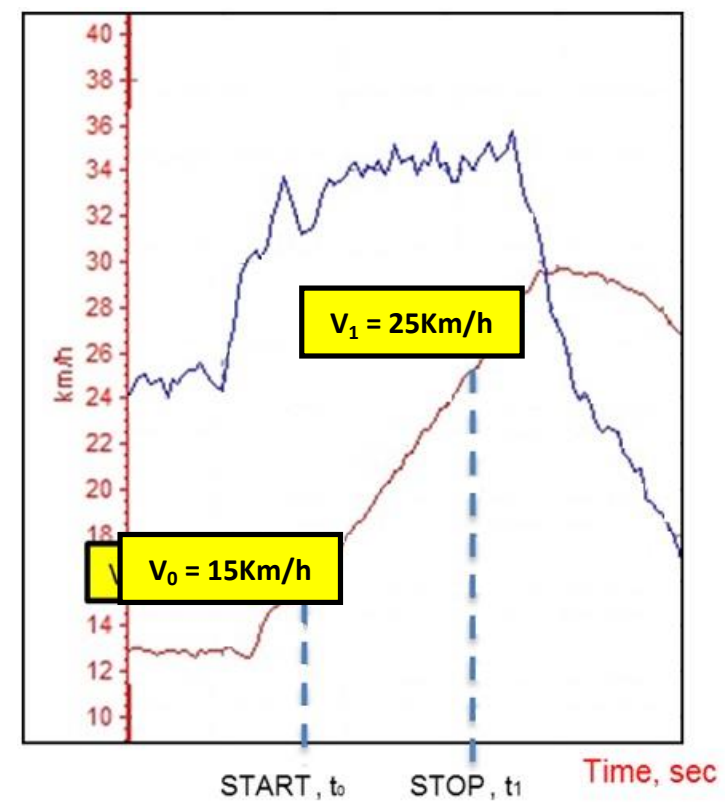

Figure 5. Acceleration and speed behavior. 
Table 5 shows the subjective drivability rating gathered from one of the test evaluators. All evaluators can differentiate the level of acceleration set by applying mechanical load to the vehicle. Although the mechanical load is varied throughout the test for each participant, the subjective rating will be based on a vehicle's acceleration data.

Table 5. Subjective drivability rating for Proton Model 1.

\begin{tabular}{cccc}
\hline Acceleration setting & $\begin{array}{c}\text { Subjective } \\
\text { Drivability Rating }\end{array}$ & $\begin{array}{c}15 \mathrm{~km} / \mathrm{h}-25 \mathrm{~km} / \mathrm{h} \\
\text { time }(\mathrm{VBOX})\end{array}$ & Acceleration \\
\hline 1 & 7.0 & 1.58 & 1.76 \\
2 & 3.0 & 3.93 & 0.71 \\
3 & 6.0 & 1.99 & 1.40 \\
4 & 7.0 & 1.57 & 1.77 \\
5 & 4.5 & 2.87 & 0.97 \\
6 & 6.0 & 2.04 & 1.36 \\
7 & 5.0 & 2.14 & 1.30 \\
8 & 7.0 & 1.58 & 1.76 \\
9 & 3.5 & 3.29 & 0.84 \\
10 & 5.5 & 2.21 & 1.26 \\
\hline
\end{tabular}

All evaluator comments were recorded during the evaluation. All agreed that subjective drivability could be differentiated by a different acceleration feel. One of evaluators felt that a rating of 10 , which is described as exceptional, is impossible to achieve even for a very good car. Based on the rating definition, 9 is defined as no deficiencies. It means that at this level, no complaints will be received from customers. The rating is given with a high confidence level that customers will accept it, and it can be considered as a unique selling point. Since the rating is given subjectively, no one dares to use it as there is no objective vehicle data to support it. The situation is similar with the lower rating definitions. A rating of 1 is described as unpredictable. This means the vehicle behaves contrary to desired behavior. In the case of low speed passing acceleration, rating 1 described as not moving at all, even during WOT operation. Normally the evaluator would consider this as a development problem instead of giving a rating of 1. In reality, the only useful ratings that can be used are from Rating 3 to 8 . This situation will limit the subjective rating correlation process. Only $60 \%$ of the plotting area can be utilized on a 0 to 10 rating scale, as per Figure 6. Minimum ( 1 and 2) and maximum (9 and 10) ratings can be interpolated only if objective data is available.

Data for subjective drivability is plotted from surveys against a vehicle's passing acceleration. Figure 6 indicates a relationship between that data, according to regression methods. In this method, the relationship between drivability rating and passing acceleration is given as;

$$
\begin{aligned}
Y=-1.0243 X^{2}+6.3746 X-1.0103 \\
Y: \text { Drivability rating } \\
X: \text { Passing acceleration }
\end{aligned}
$$




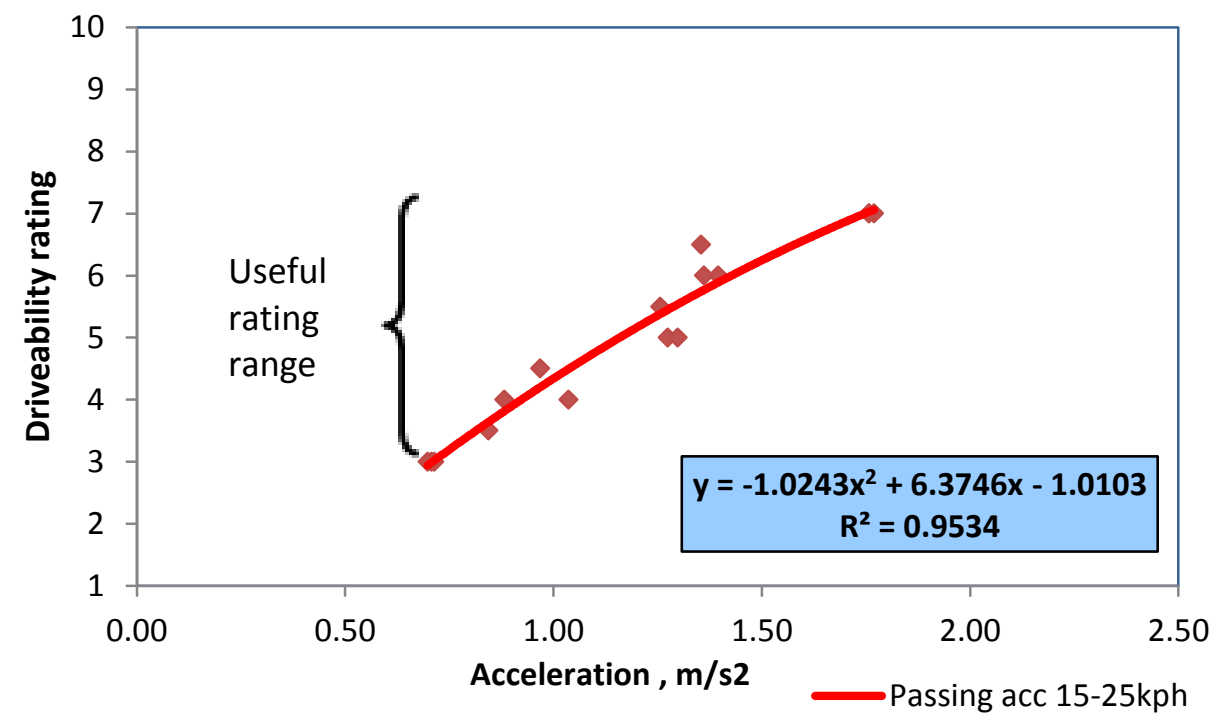

Figure 6. Drivability rating vs. acceleration

It can be concluded that there is a relationship between drivability rating and passing acceleration. Generally, a higher acceleration value will give a better drivability rating. As described earlier, there will be an acceleration value that can meet a 10 points rating, but the validity of the data may be questionable due to a lack of subjective drivability rating.

\section{VALIDATION}

To confirm the validity of the newly established drivability equation (Equation 1), a benchmarking activity is conducted. A Proton Model 2 of similar class as Proton Model 1 was selected and underwent the same evaluation procedure. From Table 6, it can be concluded that the objective drivability rating calculated from the drivability equation is in line with the subjective rating given by the test evaluators. There was only a 5\% difference in ratings, especially for lower acceleration values.

Table 6. Comparison: subjective and objective drivability.

\begin{tabular}{ccccc}
\hline \multirow{2}{*}{ Acceleration } & \multicolumn{2}{c}{ Drivability rating } & \multicolumn{2}{c}{ Difference } \\
\cline { 2 - 5 } & Subjective & Objective & Rating & $\%$ \\
\hline 1.76 & 7.0 & 7.04 & 0.04 & 0.5 \\
0.88 & 4.0 & 3.81 & -0.19 & -4.8 \\
0.71 & 3.0 & 3.00 & 0.00 & 0.0 \\
1.26 & 5.5 & 5.40 & -0.10 & -1.9 \\
0.84 & 3.5 & 3.62 & 0.12 & 3.5 \\
1.04 & 4.5 & 4.51 & 0.01 & 0.3 \\
0.97 & 4.0 & 4.21 & 0.21 & 5.0 \\
0.88 & 4.0 & 3.81 & -0.19 & -4.8 \\
1.77 & 7.0 & 7.06 & 0.06 & 0.9 \\
1.40 & 6.0 & 5.91 & -0.09 & -1.6 \\
\hline
\end{tabular}


Using VBOX Tools software as per Figure 7, the objective drivability equation (Equation 1) was successfully modeled. A data acquisition system was set to get real time data directly from the sensor. A final drivability rating will be produced immediately all conditions are met. With this system, a drivability rating can be produced in real time. All feedback for the drivability rating can be received immediately. Designers can have their own drivability assessment during initial vehicle development using the objective drivability assessment tool. All corrections in design can be performed earlier.

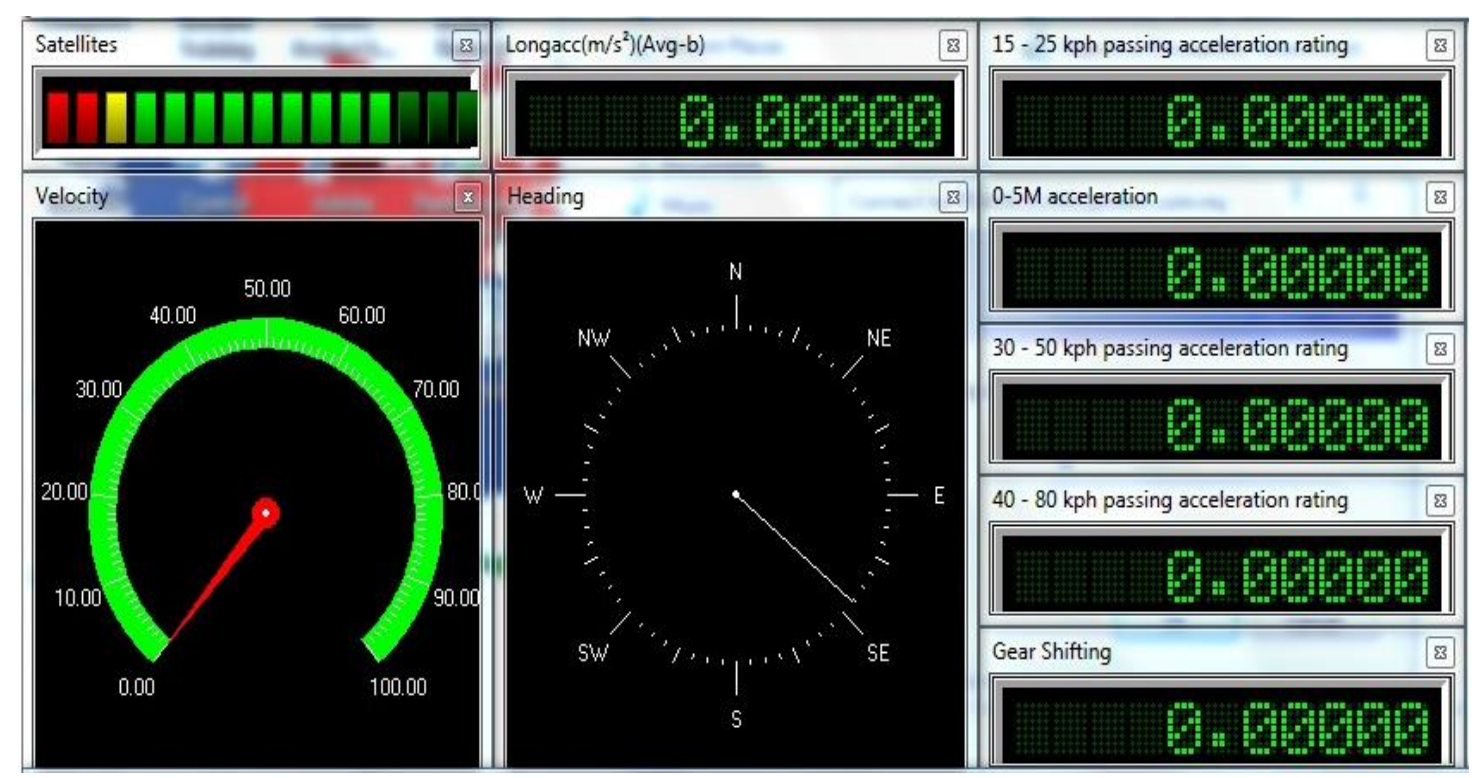

Figure 7. VBOX Tools software user interface with objective drivability rating display

\section{CONCLUSION}

An objective drivability assessment will give clear judgments about actual vehicle drivability conditions. Ratings produced will be accurate every time without depending on humans. An objective drivability assessment tool is successfully arranged using Equation (1). Real time drivability will help engineers to immediately tune ECU characteristics to achieve better ratings. Dependency on expert evaluators for producing subjective ratings can be reduced. The experts will only act as validators, for approval of the objective drivability assessment. Development timing can be shortened and all drivability issues can be detected earlier. With the establishment of the relationship between subjective feeling and vehicle data, other subjective drivability parameters can also be converted into objective assessment. Parameters such as vehicle response at any throttle percentage, initial $0-5$ meter response, overtaking performance and gear ratio evaluation are some of the subjective aspects of drivability that can be evaluated using this objective assessment method.

\section{ACKNOWLEDGEMENTS}

The authors would like to thank the Homologation and Testing department, Engineering Division, Proton for supporting the research in terms of the test vehicle and expert evaluation opinions in collecting subjective drivability ratings. 


\section{REFERENCES}

AVL List. (2000). Drivability simulation in the continuous development process. In A. L. GmbH (Ed.). Graz.

Cross, J. F., Thirard, C., Antoine, T., \& Dolcini, P. (2010). Wide BRIO benchmark and objective rating of the acceleration feeling. Automotive and railway comfort, 8.

Doray, R. E., \& Martin, E. J. (2000). Vehicle drivability - the development of an objective methodology. Paper presented at the 2000 World Congress, Michigan.

Dorey, R. E., \& Holmes, C. B. (1999). Vehicle driveability-its characterisation and measurement: SAE Technical Paper.

Neculita, C.-M., Zagury, G. J., \& Bussière, B. (2007). Passive Treatment of Acid Mine Drainage in Bioreactors using Sulfate-Reducing Bacteria. J. Environ. Qual., 36(1), 1-16. doi: 10.2134/jeq2006.0066

Palumbo, G., Amante, F., \& Andrea Ugo, A. (May 2007). Drive away quality - testing procedure for manual transmission based upon experimental analysis of customer driving. Paper presented at the Engine Expo, Turin.

Proton, P. O. N. S. B. (2009). Vehicle drivability evaluation test procedure 07-1109/A/3-00004939. Shah Alam.

SAE. (1993). Cold start and drivability procedure (Vol. J1635): Society of Automotive Engineers.

Schoeggl, P., \& Ramschak, E. (2000). Vehicle driveability assessment using neural networks for development, calibration and quality tests: SAE Technical Paper.

Schöggl, P., Koegeler, H. M., Gschweitl, K., Kokal, H., Williams, P., \& Hulak, K. (2002). Automated EMS calibration using objective driveability assessment and computer aided optimization methods: SAE Technical Paper.

Yassir, I. A. (2008). Project Eagle development cold climate test report. Unpublished Note. 